\section{SALIVARY CORTISOL LEVELS IN PREPUBERTAL CHILDREN USING INHALED CORTICOSTEROIDS WITH OR WITHOUT CONCURRENT INTRANASAL CORTICOSTEROIDS}

\section{S.M. Heijsman, A.W.A. Kamps, A. Wolthuis T.W. de Vries \\ Medical Centre Leeuwarden, Leeuwarden, The Netherlands}

Background: Inhaled corticosteroids (ICS) and intranasal steroids (INS) are frequently coadministered in children with asthma and rhinitis. In contrast to monotherapy with ICS or INS, little is known about the safety of concurrent use of topical steroids on HPA axis function in prepubertal children.

Objective: Comparison of morning salivary cortisol levels in prepubertal children using maintenance treatment with ICS with and without concurrent use of INS to a steroid naïve control group.

Methods: Cross-sectional observational study in prepubertal children (6-12 years) using ICS alone $(n=41)$ or in combination with INS $(n=22)$, compared to a control group with no steroid treatment $(n=28)$. Morning salivary cortisol levels were determined from saliva samples collected at home.

Results: The morning salivary cortisol levels of prepubertal children using ICS (median $5.1 \mathrm{nmol} / \mathrm{l}$; $95 \% \mathrm{Cl} 4.1$ to 7.6 ) or a combination of ICS and INS (4.8 nmol//; 4.6 to 8.1) were comparable, but significantly reduced compared to the steroid naïve control group (8.9 nmol/l; 8.0 to 11.3). There was no correlation between salivary cortisol level and cumulative daily dose of topical steroids.

Conclusion: Salivary cortisol levels in prepubertal children using ICS, with or without concurrent use of INS, were comparable. However, salivary cortisol levels were significantly reduced compared to steroid naïve controls, irrespective of the cumulative daily dose of topical steroids.

\section{WATER CONTENT IN INFANT STRATUM CORNEUM}

\author{
G.N. Stamatas ${ }^{1}$, J. Nikolovski ${ }^{2}$ \\ ${ }^{1}$ Johnson \& Johnson Consumer France, \\ Issy-les-Moulineaux, France, ${ }^{2}$ Johnson \& Johnson \\ Consumer Companies, Skillman, NJ, USA
}

Objective: To determine the water content of infant stratum corneum (SC) as compared to adult and identify the sources of differences.

Methods: We have acquired in vivo Raman spectra from the forearm skin of 15 infants (3-24 months) and their biological mothers. The Raman spectra were analyzed for the water content, the thickness of $\mathrm{SC}$, and the concentration of components of natural moisturization factor (NMF). We also collected skin conductance data on the forearm of 67 infants of the same age and their mothers.

Results: As previously reported conductance values and water concentration profiles are significantly higher in infant skin compared to adult. In agreement with published data from confocal microscopy, Raman data also show that infant SC is $30 \%$ thinner than adult. Even after accounting for the differences in SC thickness our data show that water content in the top layers of the SC is still significantly higher in infants compared to adults (20\% more at the top quarter of SC). NMF analysis showed that although adults have more amino acids, infants have significantly more lactate in their SC.

Conclusions: Infant SC contains more water than adult. Lactate may be responsible for at least part of this increased water content. Water stored in microrelief lines may be an additional explanation for the higher skin conductance values in infant skin.

258

HELICOBACTER PYLORI INFECTION IN ALLERGIC CHILDREN

D. Akmentina, S. Remberga,

I. Rumba-Rozenfelde, S. Limeza, R. Seske, D.D. Senaratne, I. Daugule

Faculty of Medicine, University of Latvia, Riga, Latvia

Background and aims: Studies have suggested that Helicobacter pylori (H.pylori) infection could 
be considered as a protective factor against development of allergic illnesses. The goal of our study was to compare the prevalence of $H$. pylori infection in allergic patients and children without allergy.

Methods: The total patient sample consisted of 147 children aged 1-10 yrs (mean age 5.2): 58 children had allergy diagnosis and 89 were healthy children or children with minor health problems. Parents were asked to bring a faecal samples of the child and to fill out a questionnaire (demographic data, socio-economical factors). Presence of active $\mathrm{H}$. pylori infection was detected by stool antigen test (H. pylori rapid test Coris BioConcept, Belgium ). Statistical analysis: $X^{2}$ test, log regression. Results: The total prevalence of H.pylori infection was $7 \%(10 / 147)$. In the univariate analysis H.pylori positivity was significantly lower in allergic children compared to children without allergy: $1.7 \%(1 / 58)$ vs. $10 \%$ (9/89): $p=0.048$; OR 6.4 (Cl:0.7-52). H.pylori positivity was significantly associated with a lower educational status of the mother $(p=0.01)$. In logistic regression analysis the variable having allergy did not show significant association with H.pylori infection ( $p>0.05)$.

Conclusions: Lower H.pylori prevalence observed in children with allergy could rather be associated with some other factors (previous treatment, higher living standards). However, the role of H.pylori in hygienic hypothesis and development of allergic disease should be studied further.

\section{9}

\section{EFFECTS OF TRAFFIC AIR POLLUTION ON RESPIRATORY HEALTH AND ALLERGIES IN SCHOOLCHILDREN}

\author{
W. Al Qerem, K. McGarry, L. Neshat, \\ M. Shamssain
}

Department of Pharmacy, Health and Wellbeing, University of Sunderland, Sunderland, UK

The objective of the study is to investigate gender differences in the respiratory effects of air pollution in schoolchildren. We studied 1397 schoolchildren from two locations in Egypt; Cairo city with high level of air pollution and Shbeen Al Koom in the Delta with low level of air pollution. Lung function testing was done by the Vitalograph spirometer. The Arabic version of ISAAC questionnaire was used (the International Study of Asthma and Allergies in Childhood). Air pollution measurements were collected from the Government sites in both locations. Boys in Shbeen Al Koom had significantly $(p<0.05)$ higher lung function tests than boys in Cairo. There was no significant differences in lung function tests between girls in both locations. Children in Cairo had significantly $(p<0.01)$ higher prevalence rates of asthma, rhinitis and eczema than children in Shbeen Al Koom. The prevalence rates of ever rhinitis were $6 \%$ and $3 \%$ higher in boys and girls in Cairo compared with Shbeen Al Koom, respectively. Children who developed rash less than 2 years of age were $2 \%$ and $5 \%$ higher in boys and girls in Cairo compared with Shbeen Al Koom, respectively. The prevalence of other allergic symptoms were $2-3 \%$ and $3-5 \%$ higher in boys and girls in Cairo compared with Shbeen Al Koom, respectively. The present study shows the adverse respiratory effects of exposure to traffic air pollution on schoolchildren showing gender difference. The study will help to implement strategic health intervention programmes to improve the respiratory health of children.

\section{0}

\section{ARE TYPE 1 DIABETES CHILDREN AND ADOLESCENTS AT RISK FOR AN IMPAIRED BONE MINERALIZATION?}

\author{
I. Roggen ${ }^{1}$, I. Gies ${ }^{1}$, J. Vanbesien ${ }^{1}$, O. Louis², \\ J. De Schepper ${ }^{1}$ \\ ${ }^{1}$ Pediatrics, ${ }^{2}$ Radiology, UZ Brussel, \\ Brussel, Belgium
}

Aim: Diabetic adolescents, particularly girls, often show poor metabolic control. The aim of this study is to investigate whether glycemic control (GC) during puberty has an influence on radial trabecular bone mineral density (BMD) at the final growth.

Methods: We studied 52 adolescent type 1 diabetes mellitus (DM1) (24 females) at the end of puberty. Median (range) age was 18.4 (17.2-24.8). Duration of diabetes ranged between 1.4-19.9 years (median 9.1). Trabecular BMD of the distal radius was investigated using peripheral quantitative computed tomography (XCT-2000, Stratec, Germany). Mean glycosylated hemoglobin $(\mathrm{HbA} 1 \mathrm{c})$ in the last 4 years (13.8 \pm 3.9 measurements per patient) was calculated as GC parameter. We recruited 44 healthy controls (C) (32 females; similar age range).

Results: In males DM1 and C subjects had similar height, weight, body mass index (BMI), trabecular BMD and age-adjusted trabecular BMD values 